\title{
A Monge-Ampère equation in conformal geometry
}

\author{
MATTheW J. GURSKY
}

\begin{abstract}
We consider the Monge-Ampère-type equation $\operatorname{det}(A+\lambda g)=$ const ., where $A$ is the Schouten tensor of a conformally related metric and $\lambda>0$ is a suitably chosen constant. When the scalar curvature is non-positive we give necessary and sufficient conditions for the existence of solutions. When the scalar curvature is positive and the first Betti number of the manifold is non-zero we also establish existence. Moreover, by adapting a construction of Schoen, we show that solutions are in general not unique.
\end{abstract}

Mathematics Subject Classification (2000): 53A30.

\section{Introduction}

Fully nonlinear equations arise naturally in differential geometry when studying equations of prescribed curvature, for example of hypersurfaces in Euclidean space or Riemannian manifolds under conformal changes of metric. Typically one imposes a constraint on the solution space, often referred to as admissibility, in order to guarantee that the equations are elliptic. For example, when studying the MongeAmpère equation one considers convex solutions.

While technically advantageous this condition can sometimes be geometrically unnatural, or at least overly restrictive. To give an example which is relevant to the main results of the present paper, consider the so-called $k$-Yamabe problem: given a compact Riemannian manifold $\left(M^{n}, g\right)$, find a conformal metric $\widehat{g}=e^{-2 u} g \in[g]$ so that

$$
\sigma_{k}(\widehat{A})=\text { const ., }
$$

where $\widehat{A}=A(\widehat{g})$ is the Schouten tensor of the metric $\widehat{g}$, and $\sigma_{k}(\cdot)$ is the $k$-th elementary symmetric polynomial applied to the eigenvalues of $A(\widehat{g})$. Equation (1.1) is equivalent to the fully nonlinear PDE

$$
\sigma_{k}\left(A(g)+\nabla^{2} u+d u \otimes d u-\frac{1}{2}|d u|^{2} g\right)=c e^{-2 k u} .
$$

Research supported in part by NSF Grant DMS-0500538.

Received July 31, 2007; accepted January 15, 2008. 
This equation is elliptic if the Schouten tensor $A=A(g)$ of $g$ is $k$-convex at each point and $c>0$. When this holds we say that the metric $g$ is admissible, or $k$ admissible. There is now an extensive literature on the $k$-Yamabe problem, and we refer the reader to the recent survey in [13].

In low dimensions admissibility is a very strong condition: for example, if $\left(M^{3}, g\right)$ is admissible then $g$ has positive sectional curvature (see [4, Proposition 1.1]). This excludes the possibility of even studying, let alone solving, the $k$ Yamabe problem on many smooth 3-manifolds. For example, the product manifold $M^{3}=S^{2} \times S^{1}$ does not admit an admissible metric. Indeed, by Hamilton's theorem the only 3-manifolds which admit admissible metrics are quotients of the sphere.

Another technical anomaly of the $k$-Yamabe problem is the lack of any existence theory for manifolds of negative curvature. Equation (1.2) is also elliptic if $(-A)$ is $k$-convex and $(-1)^{k} c>0$; i.e., negative admissible metrics. However, as in the positive case, this is a strong geometric condition in low dimensions. Analytically, the situation is even worse: when $k \geq 2$, there are counterexamples to local $C^{2}$-estimates for solutions (see [11, Section 3]), rendering the usual existencefrom-estimates approach ineffectual.

Our goal in this paper is propose a generalization of the $k$-Yamabe problem which retains some of the geometric flavor of the original, while bypassing many of the non-essential technical deficiencies. To this end, let $\left(M^{n}, g\right)$ be a compact Riemannian manifold without boundary. Our goal is to find a conformal metric $\widehat{g}=e^{-2 u} g$ such that

$$
\operatorname{det}(\widehat{A}+\lambda \widehat{g})^{1 / n}=\mu
$$

where $\lambda, \mu>0$ are constants. This is equivalent to solving the fully nonlinear PDE

$$
\operatorname{det}\left(A+\lambda e^{-2 u} g+\nabla^{2} u+d u \otimes d u-\frac{1}{2}|d u|^{2} g\right)^{1 / n}=\mu e^{-2 u}
$$

Here are some reasons for viewing (1.3) as a natural generalization of (1.1):

- The equation (1.3) is conformally invariant: i.e., if $\left(M^{n}, \widehat{g}\right)$ is a solution of (1.3) and $\varphi: M^{n} \rightarrow M^{n}$ is a conformal map, then $\varphi^{*} \widehat{g}$ is also a solution.

- Equation (1.3) can be studied on any smooth manifold. Indeed, for reasons of scaling to be discussed below the choice of $\lambda>0$ is essentially arbitrary, and for an appropriate scaling of the background metric our equation will always be elliptic.

- The term $\lambda \widehat{g}$ is only a zeroth-order perturbation of the metric. In particular, we have not "gained" ellipticity by changing the highest order nonlinearity in the equation, as is sometimes done in the $k$-Yamabe problem (see for example $[5,6]$ ).

- The semilinear version of (1.3) reduces to the usual Yamabe problem. That is, the equation

$$
\sigma_{1}(\widehat{A}+\lambda \widehat{g})=\mu
$$


is equivalent to

$$
R(\widehat{g})=\text { const } .
$$

- Certain geometric structures are preserved by the addition of the lower order term $\lambda \widehat{g}$. For example, if $\widehat{g}$ is Einstein then $\widehat{A}$ is pure trace, and the same is true for $\widehat{A}+\lambda \widehat{g}$. Or, if $\widehat{g}$ is locally conformally flat then $\widehat{A}$ is a Codazzi tensor, and the same is true for $\widehat{A}+\lambda \widehat{g}$.

Before stating the main existence results for equation (1.3) we need to make a definition:

Definition 1.1. We say that the metric $g$ is $\lambda$-admissible if $\lambda>0$ and

$$
A+\lambda g>0,
$$

where $A=A_{g}$ is the Schouten tensor of $g$.

Of course, every Riemannian metric is $\lambda$-admissible for some $\lambda \gg 0$ sufficiently large. Moreover, given $\lambda>0$ and a metric $g$, by a simple homothety we can make the new metric (which is conformal to $g$ ) $\lambda$-admissible. Actually, in this context it is worth pointing out how equation (1.3) scales: If $\widehat{g}$ is a solution of (1.3), then $h=t^{2} \widehat{g}$ is a solution of

$$
\operatorname{det}\left(A(h)+\lambda_{t} h\right)^{1 / n}=\mu_{t},
$$

where

$$
\lambda_{t}=t^{-2} \lambda, \quad \mu_{t}=t^{-2} \mu .
$$

In particular, $\lambda$ and $\mu$ scale in the same way. As we shall see, the conformal structure imposes certain necessary conditions on the ratio $\lambda / \mu$.

For our first existence result we assume the conformal class of $g$ admits a metric of non-positive scalar curvature. As we show in Section 2, in this case a necessary condition for the existence of a $\lambda$-admissible solution of (1.3) is that $\lambda \geq$ $\mu$; moreover, equality holds if and only if $g$ is Ricci-flat and $\widehat{g}$ is a homothety of $g$. This condition is also sufficient:

Theorem 1.2. Let $\left(M^{n}, g\right)$ be a smooth, closed Riemannian manifold of dimension $n \geq 3$. Suppose $g$ is $\lambda$-admissible for some $\lambda>0$.

If the scalar curvature of $g$ is negative; or if $g$ is scalar-flat but not Ricci-flat, then for each $0<\mu<\lambda$ there is a $\lambda$-admissible conformal metric $\widehat{g}=e^{-2 u} g$ satisfying

$$
\operatorname{det}(\widehat{A}+\lambda \widehat{g})^{1 / n}=\mu
$$

Moreover, the set of all $\lambda$-admissible solutions of (1.6) is compact in $C^{k}\left(M^{n}\right)$, for any $k \geq 0$. 
Somewhat surprisingly, a solution of (1.6) may not be unique. In Section 6 we show how a construction due to R. Schoen can be adapted to our equation to construct multiple solutions on certain product manifolds.

In view of the scaling properties described above we can state our existence result in more general terms:

Corollary 1.3. If $[g]$ admits a metric of negative scalar curvature, or a metric which is scalar-flat but non-Ricci-flat, then there is a $\lambda$-admissible solution $\widehat{g} \in[g]$ of (1.6) if and only if $0<\mu<\lambda$.

Proof. Suppose $g_{0} \in[g]$ has negative scalar curvature, or is scalar-flat but not Ricci-flat. Given $\lambda>0$, we can always choose $t \gg 1$ so that $h=t^{2} g_{0}$ is $\lambda$ admissible. The existence of $\widehat{g} \in[g]$ then follows from the preceding theorem. For necessity, see Proposition 2.1 in Section 2.

\subsection{The case of positive scalar curvature}

If the conformal class of $g$ is positive ${ }^{1}$ then there may be no relationship between $\lambda$ and $\mu$, and the existence theory seems more complicated (see Section 2.2). Moreover, the conformal invariance of equation (1.3) allows the possibility of bubbling, and consequently the failure of a priori estimates for solutions. Of course, bubbles are spherical appendages, and the Schouten tensor of the sphere is strictly positive. Therefore, if our conformal class does not admit a metric whose Schouten tensor is "mostly" positive it should be possible-in principle at least-to rule out bubbling. This heuristic argument is made precise in Section 5. Since the statement is rather technical, we only state a corollary of the more general result (Theorem 5.3):

Theorem 1.4. Suppose $M^{n}$ is a smooth, closed manifold of dimension $n \geq 3$ whose first Betti number $b_{1}\left(M^{n}\right) \neq 0$. Then given any positive conformal class of metrics [g] on $M^{n}$, and any pair of numbers $0<\mu<\lambda$, there is a $\lambda$-admissible solution $\widehat{g} \in[g]$ of (1.3).

Remark. Of course, if $[g]$ is a negative conformal class on $M^{n}$, or a zero conformal class but without a Ricci-flat metric, then existence follows from Theorem 1.2.

This result is a striking contrast to the $k$-Yamabe problem. For example, any oriented four-manifold with $\chi\left(M^{4}\right) \leq 0$ cannot admit an admissible (or negative admissible) metric, but since $b_{1}\left(M^{4}\right)>0$, every conformal class admits a solution of (1.3) for an appropriate choice of $\lambda, \mu$.

\subsection{Other symmetric functions}

One can also consider more general versions of (1.3), such as

$$
\sigma_{k}(\widehat{A}+\lambda \widehat{g})^{1 / k}=\mu,
$$

${ }^{1}$ By a positive, negative or zero conformal class we will mean a conformal class which admits a metric of strictly positive, strictly negative, or identically zero scalar curvature. 
which is equivalent to

$$
\sigma_{k}\left(A+\lambda e^{-2 u} g+\nabla^{2} u+d u \otimes d u-\frac{1}{2}|d u|^{2} g\right)^{1 / k}=\mu e^{-2 u} .
$$

Additionally, one could consider equations involving the Ricci tensor in place of the Schouten tensor; for example,

$$
\operatorname{det}(\widehat{\operatorname{Ric}}+\lambda \widehat{g})^{1 / n}=\mu
$$

Many of the techniques of this paper apply to these equations as well. In particular, one can prove an existence result analogous to Theorem 1.2 with the obvious modifications. However, to simplify the exposition we will restrict our attention to the determinant equation, and with the Schouten tensor.

\subsection{An application}

In a subsequent paper we plan to pursue various geometric applications of the equations considered here. However, since it follows in a rather straightforward manner from the proof of Theorem 1.2, in the final section of the paper we present a small application. This application was inspired by recent work of Guan-Wang [8], who proved that any manifold of strictly negative Ricci curvature is conformal to one with $\rho_{\min }[$ Ric $]=-(n-1)$, where $\rho_{\min }[\cdot]$ denotes the smallest eigenvalue. Moreover, the regularity of the conformal factor is $C^{2, \alpha}$ for some $\alpha>0$. Using our main existence result Theorem 1.2 we can prove an analogous result under much weaker assumptions, but with weaker regularity conclusions:

Theorem 1.5. If $g$ has negative scalar curvature, or if $g$ is scalar-flat but not Ricciflat, then there is a $C^{1,1}$-conformal metric $\widehat{g}=e^{-2 u} g$ with

$$
\rho_{\min }[\widehat{A}]=-1 / 2 \text { a.e. }
$$

The loss of regularity is due to the loss of uniform ellipticity of (1.4) as $\mu \rightarrow 0$. As indicated above, one can prove a corresponding result for the Ricci curvature. Note that the eigenvalues of the Schouten tensor on hyperbolic space are all $-1 / 2$.

As a final remark, we point out that one could alternatively subtract a multiple of the metric and consider negative $\lambda$-admissble metrics; i.e., metrics for which

$$
A-\lambda g<0
$$

and attempt to solve

$$
\operatorname{det}(\widehat{A}-\lambda \widehat{g})=(-1)^{n} \mu^{n}
$$

for some $\mu>0$. Unfortunately, this equation behaves much worse than (1.3) and seems to share many of the same difficulties with the negative cone equation for 
the $k$-Yamabe problem. Nevertheless, some of the techniques in this paper are still applicable.

ACKNOWLEDGEMENTS. The research for this paper was carried out while the author was visiting the Institut des Hautes Études Scientifique. The author gratefully acknowledges the support, both material and intellectual, of the Institute.

\section{2. $\lambda$ - and weak admissibility}

In this section we prove some facts about $\lambda$-admissible conformal metrics $\widehat{g}=$ $e^{-2 u} g$ satisfying

$$
\operatorname{det}(\widehat{A}+\lambda \widehat{g})^{1 / n}=\mu,
$$

where $\mu>0$ is a constant. As we shall see, the conformal structure may impose necessary conditions on the value of $\mu$. To simplify the exposition we will consider the case of non-positive and positive scalar curvature separately.

\subsection{Non-positive scalar curvature}

The case of non-positive scalar curvature is easily described:

Proposition 2.1. Suppose $\widehat{g}=e^{-2 u} g$ is a $\lambda$-admissible solution of (2.1).

(i) If $\sigma_{1}\left(A_{g}\right)<0$, then $\lambda>\mu$.

(ii) If $\sigma_{1}\left(A_{g}\right) \equiv 0$, then $\lambda \geq \mu$, and $\lambda=\mu$ if and only if $g$ is Ricci-flat and $u$ is constant.

Proof. Both statements are consequences of the Arithmetic-Geometric-Mean inequality along with the maximum principle. First, rewriting equation (2.1) in terms of $u$ we have

$$
\operatorname{det}\left(A+\lambda e^{-2 u} g+\nabla^{2} u+d u \otimes d u-\frac{1}{2}|d u|^{2} g\right)^{1 / n}=\mu e^{-2 u} .
$$

By the A-G-M inequality,

$$
\begin{aligned}
\mu e^{-2 u} & \leq \frac{1}{n} \sigma_{1}\left(A+\lambda e^{-2 u} g+\nabla^{2} u+d u \otimes d u-\frac{1}{2}|d u|^{2} g\right) \\
& =\frac{1}{n} \sigma_{1}(A)+\lambda e^{-2 u}+\frac{1}{n} \Delta u-\left(\frac{n-2}{2 n}\right)|d u|^{2},
\end{aligned}
$$


hence

$$
(\mu-\lambda) e^{-2 u} \leq \frac{1}{n} \sigma_{1}(A)+\frac{1}{n} \Delta u .
$$

Let $x_{1}$ be a point at which $u$ attains its maximum; then

$$
(\mu-\lambda) e^{-2 u\left(x_{1}\right)} \leq \frac{1}{n} \sigma_{1}(A)\left(x_{1}\right)
$$

Therefore, if $\sigma_{1}(A)<0$ then $\mu<\lambda$, while if $\sigma_{1}(A)=0$ we have $\mu \leq \lambda$. Moreover, if $\sigma_{1}(A)=0$ and $\mu=\lambda$, then by (2.3) we see that $u$ must be subharmonic, hence constant. Moreover, we have equality in the A-G-M inequality, so $A$ is diagonal and $g$ is Ricci-flat.

\subsection{Positive scalar curvature}

In general, when the scalar curvature of $g$ is positive there is no obvious relationship between $\lambda$ and $\mu$. For example, let $M^{n}=S^{n-1} \times S^{1}$ endowed with the product metric $g$. Since the Schouten tensor can be diagonalized at each point as

$$
A=\left(\begin{array}{cccc}
1 / 2 & & & \\
& \ddots & & \\
& & 1 / 2 & \\
& & & -1 / 2
\end{array}\right)
$$

it follows that $\left(M^{n}, g\right)$ is $\lambda$-admissible for all $\lambda>1 / 2$. Take $\lambda=1 / 2+\delta$, where $\delta>0$; then

$$
\mu=\operatorname{det}(A+\lambda g)^{1 / n}=\delta^{\frac{1}{n}}(1+\delta)^{\frac{(n-1)}{n}} .
$$

Consequently, if $\delta>0$ is small, then $\mu<\lambda$, while if $\delta>>1$, then $\mu>\lambda$.

On the other hand if we impose a "weak admissibility" condition on the conformal class of $g$ then one can say something about $\mu$. To this end we make the following definition:

Definition 2.2. We say that $w \in \mathcal{W}_{n}([g])$ if $w$ is continuous, and there is a sequence of smooth functions $\left\{w_{i}\right\}$ converging uniformly to $w$ with the following property: Given $\delta>0$, there is a $N=N(\delta)$ such that

$$
\begin{aligned}
A\left(e^{-2 w_{i}} g\right)+\delta e^{-2 w_{i}} g= & A(g)+\nabla^{2} w_{i} \\
& +d w_{i} \otimes d w_{i}-\frac{1}{2}\left|d w_{i}\right|^{2} g+\delta e^{-2 w_{i}} g>0
\end{aligned}
$$

for all $i \geq N$.

A function $w \in \mathcal{W}_{n}([g])$ will be called weakly admissible. 
To provide some motivation for this definition, first recall that the set of $k$ admissible metrics in the conformal class of $g$ is usually denoted $\mathcal{C}_{k}([g])$. In particular, when $k=n$ this is the set of $n$-admissible metrics-i.e., metrics whose Schouten tensors are positive definite. Clearly,

$$
\mathcal{C}_{n}([g]) \subseteq \mathcal{W}_{n}([g])
$$

since if $\widetilde{g}=e^{-2 w} g \in \mathcal{C}_{n}$ then we can take $w_{i}=w$ for each $i$ in Definition 2.2. One can extend this definition to other symmetric functions in the obvious way: $w \in \mathcal{W}_{k}([g])$ iff $w$ is continuous, and there is a sequence of smooth functions $\left\{w_{i}\right\}$ converging uniformly to $w$ such that for each $\delta>0$, there is a $N=N(\delta)$ with

$$
A\left(e^{-2 w_{i}} g\right)+\delta e^{-2 w_{i}} g \in \Gamma_{k}^{+}
$$

for all $i \geq N$. Again, we have the obvious inclusion

$$
\mathcal{C}_{k}([g]) \subseteq \mathcal{W}_{k}([g])
$$

The precise relationship between $\mathcal{W}_{k}$ and $\mathcal{C}_{k}$ seems to be a delicate question. For example, consider the product manifold

$$
M^{4}=S^{2}(1) \times S^{2}(\sqrt{2}),
$$

whose Schouten tensor is given by

$$
A=\left(\begin{array}{cccc}
1 / 4 & & & \\
& 1 / 4 & & \\
& & 0 & \\
& & & 0
\end{array}\right) .
$$

Clearly $\mathcal{W}_{4} \neq \emptyset$. However, since $\operatorname{det}(A) \equiv 0$, by the maximum principle it follows that $\mathcal{C}_{4}=\emptyset$ (see [7, Lemma 3]).

The preceding definitions may appear somewhat technical but will be seen to be natural in view of the estimates of Section 4. Here, we just want to observe that the existence of weakly admissible functions imposes a necessary condition on $\lambda / \mu$ :

Proposition 2.3. Suppose $\mathcal{W}_{n}([g]) \neq \emptyset$. Let $\widehat{g}=e^{-2 u} g$ be a (smooth), $\lambda$-admissible solution of (2.1). Then $\lambda \leq \mu$.

Proof. Since $\mathcal{W}_{n}([g]) \neq \varnothing$ there is a uniformly convergent sequence of smooth functions $\left\{w_{i}\right\}$ satisfying the conditions in Definition 2.2. Denote

$$
\begin{aligned}
& u_{i}=u-w_{i}, \\
& g_{i}=e^{-2 w_{i}} g .
\end{aligned}
$$


Then $\widehat{g}=e^{-2 u_{i}} g_{i}$, and we can rewrite equation (2.2) as

$$
\operatorname{det}\left(A\left(g_{i}\right)+\lambda e^{-2 u_{i}} g_{i}+\nabla_{g_{i}}^{2} u_{i}+d u_{i} \otimes d u_{i}-\frac{1}{2}\left|d u_{i}\right|^{2} g_{i}\right)^{1 / n}=\mu e^{-2 u_{i}}
$$

Let $x_{0}$ be a point at which $u_{i}$ attains its minimum; then $d u_{i}\left(x_{0}\right)=0$, so

$$
\operatorname{det}\left(A\left(g_{i}\right)\left(x_{0}\right)+\lambda e^{-2 u_{i}\left(x_{0}\right)} g_{i}\left(x_{0}\right)+\nabla_{g_{i}}^{2} u\left(x_{0}\right)\right)^{1 / n}=\mu e^{-2 u_{i}\left(x_{0}\right)} .
$$

Since $\left\{u_{i}\right\}$ is uniformly bounded, we can choose a number $\delta>0$ small enough so that

$$
\lambda e^{-2 u_{i}\left(x_{0}\right)}>\delta
$$

for all $i$. Then, by the properties of the sequence $\left\{w_{i}\right\}$ we can also assume that

$$
A\left(g_{i}\right)\left(x_{0}\right)+\delta g_{i}\left(x_{0}\right)>0
$$

for $i$ large. Therefore,

$$
\begin{aligned}
& \operatorname{det}\left(\left[A\left(g_{i}\right)\left(x_{0}\right)+\delta g_{i}\left(x_{0}\right)\right]+\left[\lambda e^{-2 u_{i}\left(x_{0}\right)}-\delta\right] g_{i}\left(x_{0}\right)+\nabla_{g_{i}}^{2} u\left(x_{0}\right)\right)^{1 / n} \\
& =\mu e^{-2 u_{i}\left(x_{0}\right)} .
\end{aligned}
$$

Using (2.8), the concavity of the determinant, and the fact that $\nabla_{g_{i}}^{2} u\left(x_{0}\right) \geq 0$, from (2.9) we conclude

$$
\lambda e^{-2 u_{i}\left(x_{0}\right)}-\delta \leq \mu e^{-2 u_{i}\left(x_{0}\right)}
$$

for all $i$ large enough. Since $\delta$ was arbitrary and $u_{i}\left(x_{0}\right)$ is bounded, we see that $\lambda \leq \mu$.

To summarize our results so far: if $[g]$ admits a metric of non-positive scalar curvature then $\lambda \geq \mu$, while if $[g]$ admits a weakly admissible function then $\lambda \leq \mu$. These are necessary conditions: they show that certain assumptions on the conformal class imply certain inequalities between $\lambda$ and $\mu$. The final result of this section is in the opposite direction. It says that a certain assumption on the ratio $\lambda / \mu \mathrm{im}$ plies that positivity of the Ricci curvature. Therefore, it provides an obstruction to solving (2.1) in certain cases when $\lambda / \mu$ is small.

Lemma 2.4. Suppose $\left(M^{n}, g\right)$ is a $\lambda$-admissible metric satisfying

$$
\operatorname{det}(A+\lambda g)^{1 / n}=\mu \text {. }
$$

If

$$
\lambda<\frac{n}{2(n-1)} \mu,
$$

then $g$ has positive Ricci curvature:

$$
\text { Ric } \geq \frac{1}{n}\left[\mu-2\left(\frac{n-1}{n}\right) \lambda\right] g .
$$


Proof. By definition,

$$
\text { Ric }=(n-2) A+\sigma_{1}(A) g .
$$

By the A-G-M inequality,

$$
\begin{aligned}
\mu & \leq \frac{1}{n} \sigma_{1}(A+\lambda g) \\
& =\frac{1}{n} \sigma_{1}(A)+\lambda,
\end{aligned}
$$

hence

$$
\sigma_{1}(A) \geq n(\mu-\lambda)
$$

Substituting this into (2.12),

$$
\text { Ric } \geq(n-2) A+n(\mu-\lambda) g .
$$

Since $g$ is $\lambda$-admissible,

$$
\begin{aligned}
\text { Ric } & \geq(n-2) A+n(\mu-\lambda) g \\
& =(n-2)(A+\lambda g)+(n \mu-2(n-1) \lambda) g \\
& >\frac{1}{n}\left[\mu-2\left(\frac{n-1}{n}\right) \lambda\right] g,
\end{aligned}
$$

as claimed.

\section{Preliminary estimates}

In this section we give an overview of the strategy and provide technical results for proving the main Theorem 1.2. It is important to emphasize that these results are independent of the sign of the scalar curvature, and are valid as long $\left(M^{n}, g\right)$ is $\lambda$-admissible and $\mu$ satisfies

$$
0<\mu<\lambda \text {. }
$$

To begin, define $f_{0} \in C^{\infty}\left(M^{n}\right)$ by

$$
\operatorname{det}(A+\lambda g)^{1 / n}=f_{0}(x)>0 .
$$

The goal is to use the continuity method to prove the existence of $u \in C^{4, \alpha}$ satisfying

$$
\operatorname{det}\left(A+\lambda e^{-2 u} g+\nabla^{2} u+d u \otimes d u-\frac{1}{2}|d u|^{2} g\right)=\mu e^{-2 u} .
$$


To this end, for $t \in[0,1]$ consider the operator

$$
\begin{aligned}
\Psi_{t}[u]= & \operatorname{det}\left(A+\lambda e^{-2 u} g+\nabla^{2} u+d u \otimes d u-\frac{1}{2}|d u|^{2} g\right)^{1 / n} \\
& -t \mu e^{-2 u}-(1-t) f_{0}(x) .
\end{aligned}
$$

Note that $u \in C^{2}$ satisfies $\Psi_{t}[u]=0$ if and only if $u$ is a solution of

$$
\begin{aligned}
& \operatorname{det}\left(A+\lambda e^{-2 u} g+\nabla^{2} u+d u \otimes d u-\frac{1}{2}|d u|^{2} g\right)^{1 / n} \\
& =t \mu e^{-2 u}+(1-t) f_{0}(x)
\end{aligned}
$$

Define

$$
\mathfrak{S}=\left\{t \in[0,1] \mid \exists \lambda-\text { admissible } \widehat{g}=e^{-2 u} g, u \in C^{4, \alpha} \text {, with } \Psi_{t}[u]=0\right\} .
$$

Since $\Psi_{0}[0]=0$, we see that $\mathfrak{S} \neq \emptyset$.

Proposition 3.1. S is open in $[0,1]$.

Proof. Denote

$$
\begin{aligned}
W & =A+\lambda e^{-2 u} g+\nabla^{2} u+d u \otimes d u-\frac{1}{2}|d u|^{2} g, \\
F(W) & =\operatorname{det}(W)^{1 / n} \\
F^{i j} & =\frac{\partial F}{\partial W_{i j}} .
\end{aligned}
$$

With this notation, we compute the linearization of $\Psi_{t}$ :

$$
\begin{aligned}
\Psi_{t}[u] \psi= & \left.\frac{d}{d s} \Psi_{t}[u+s \varphi]\right|_{s=0} \\
= & F^{i j}\left\{-2 \lambda e^{-2 u} \varphi g_{i j}+\nabla_{i} \nabla_{j} \varphi+\nabla_{i} \varphi \nabla_{j} u+\nabla_{i} u \nabla_{j} \varphi-\langle\nabla u, \nabla \varphi\rangle g_{i j}\right\} \\
& +2 t \mu e^{-2 u} \varphi \\
= & F^{i j}\left\{\nabla_{i} \nabla_{j} \varphi+\nabla_{i} \varphi \nabla_{j} u+\nabla_{i} u \nabla_{j} \varphi-\langle\nabla u, \nabla \varphi\rangle g_{i j}\right\} \\
& +2 e^{-2 u}\left(-\lambda F^{k k}+t \mu\right) \varphi \\
= & a^{i j}(W) \nabla_{i} \nabla_{j} \varphi+b^{k}(W) \nabla_{k} \varphi+c(x) \varphi
\end{aligned}
$$

where $a^{i j}>0$ and $c(x)$ is given by

$$
c(x)=2 e^{-2 u}\left(-\lambda F^{k k}+t \mu\right) .
$$


By elementary properties of the determinant,

$$
F^{k k} \geq 1
$$

and hence

$$
\begin{aligned}
c(x) & \leq 2 e^{-2 u}(-\lambda+t \mu) \\
& \leq 2 e^{-2 u}(\mu-\lambda) \\
& <0 .
\end{aligned}
$$

By the maximum principle the linearization of $\Psi_{t}$ is surjective, and the openness of $\mathfrak{S}$ follows from the implicit function theorem.

To prove the closedness of $\mathfrak{S}$ we first need to derive upper and lower bounds for solutions of $\Psi_{t}[u]=0$. We begin with the lower bound:

Proposition 3.2. Suppose $u \in C^{2}$ is a solution of (3.3) with $t \in[0,1]$. Then there is a constant $C=C(\mathrm{~g})>0$ such that

$$
u \geq \frac{1}{2} \log (\lambda-\mu)-C .
$$

Proof. Let $x_{0} \in M^{n}$ be a point at which $u$ attains its minimum; then $\nabla^{2} u\left(x_{0}\right) \geq 0$. Since $d u\left(x_{0}\right)=0$, by (3.3) we have

$$
\operatorname{det}\left(A\left(x_{0}\right)+\lambda e^{-2 u\left(x_{0}\right)} g+\nabla^{2} u\left(x_{0}\right)\right)^{1 / n}=t \mu e^{-2 u\left(x_{0}\right)}+(1-t) f_{0}\left(x_{0}\right) .
$$

Let

$$
a_{0}=\max \left\{0,-\rho_{\min }\left[A\left(x_{0}\right)\right]\right\},
$$

where $\rho_{\min }\left[A\left(x_{0}\right)\right]$ denotes smallest eigenvalue of $A\left(x_{0}\right)$. Now write

$$
\begin{aligned}
A\left(x_{0}\right)+\lambda e^{-2 u\left(x_{0}\right)} g+\nabla^{2} u\left(x_{0}\right)= & \left(A+a_{0} g\right)\left(x_{0}\right)+\left(\lambda e^{-2 u\left(x_{0}\right)}-a_{0}\right) g\left(x_{0}\right) \\
& +\nabla^{2} u\left(x_{0}\right) .
\end{aligned}
$$

First, suppose

$$
\lambda e^{-2 u\left(x_{0}\right)}-a_{0} \leq 0
$$

Then

$$
\begin{aligned}
\min u & =u\left(x_{0}\right) \geq \frac{1}{2} \log \lambda-\frac{1}{2} \log a_{0} \\
& \geq \frac{1}{2} \log (\lambda-\mu)-C,
\end{aligned}
$$

as claimed. 
If (3.8) does not hold, then each of the three terms on the right-hand side of (3.7) is positive definite. Therefore, by the concavity of (det) ${ }^{1 / n}$ and equation (3.6) we get

$$
\operatorname{det}\left(A+a_{0} g\right)^{1 / n}\left(x_{0}\right)+\left(\lambda e^{-2 u\left(x_{0}\right)}-a_{0}\right) \leq t \mu e^{-2 u\left(x_{0}\right)}+(1-t) f_{0}\left(x_{0}\right) .
$$

Hence,

$$
(\lambda-t \mu) e^{-2 u\left(x_{0}\right)} \leq a_{0}+(1-t) f_{0}\left(x_{0}\right),
$$

which implies (3.5).

Having established an a priori lower bound for solutions of (3.3), we can now appeal to the $C^{2}$-estimates in the appendix to conclude

Proposition 3.3. Suppose $u \in C^{4}$ is a solution of (3.3) with $t \in[0,1]$. Then there is a constant $C=C(\mathrm{~g})$, independent of $t$, such that

$$
\max _{M^{n}}\left[|\nabla u|^{2}+\left|\nabla^{2} u\right|\right] \leq C .
$$

To prove an upper bound for solutions we will need to make an assumption about the conformal class of $g$. For example, when the scalar curvature of $g$ is nonpositive an upper bound is relatively easy to prove; this will be explained in the next section. The case of positive scalar curvature (Theorem 5.3) is discussed in Section 5 .

\section{The proof of Theorem 1.2}

If one scrutinizes the proof of Proposition 2.1 (especially inequality (2.4)), it is easy to see that a solutions of (3.3) are bounded above when the scalar curvature of $g$ is negative:

Proposition 4.1. Suppose $u \in C^{2}$ is a solution of (3.3) with $t \in[0,1]$. If $\sigma_{1}(A) \leq$ $-\epsilon_{0}<0$, then there is a constant $C=C\left(n, f, g, \epsilon_{0}\right)>0$ such that

$$
u \leq \frac{1}{2} \log (\lambda-\mu)+C .
$$

Proof. Let $x_{1}$ be a point at which $u$ attains its maximum. Since $\nabla^{2} u\left(x_{1}\right) \leq 0$, by (3.3) and the A-G-M inequality

$$
\begin{aligned}
t \mu e^{-2 u\left(x_{1}\right)}+(1-t) f_{0}\left(x_{1}\right) & =\operatorname{det}\left(A\left(x_{1}\right)+\lambda e^{-2 u\left(x_{1}\right)} g+\nabla^{2} u\left(x_{1}\right)\right)^{1 / n} \\
& \leq \frac{1}{n} \sigma_{1}\left(A\left(x_{1}\right)+\lambda e^{-2 u\left(x_{1}\right)} g+\nabla^{2} u\left(x_{1}\right)\right) \\
& \leq \frac{1}{n} \sigma_{1}(A)\left(x_{1}\right)+\lambda e^{-2 u\left(x_{1}\right)} .
\end{aligned}
$$


Therefore,

$$
(\lambda-t \mu) e^{-2 u\left(x_{1}\right)} \geq-\frac{1}{n} \sigma_{1}(A)\left(x_{1}\right) .
$$

Combining the results of Propositions 4.1 and 3.2 we have

Corollary 4.2. Suppose $u \in C^{2}$ is a solution of (3.3) with $t \in[0,1]$. If $\sigma_{1}(A) \leq$ $-\epsilon_{0}<0$, then there is a constant $C=C\left(n, f, g, \epsilon_{0}\right)>0$ such that

$$
\frac{1}{2} \log (\lambda-\mu)-C \leq u \leq \frac{1}{2} \log (\lambda-\mu)+C .
$$

When $g$ is scalar-flat then the estimate (4.2) is useless, and we need to argue more indirectly:

Proposition 4.3. Suppose $u \in C^{2}$ is a solution of (3.3) with $t \in[0,1]$. If $\sigma_{1}(A) \equiv$ 0 but $g$ is not Ricci-flat, then there is a constant $C=C(g)$, independent of $t$, such that

$$
u \leq C
$$

Proof. Assume to the contrary that we have a sequence of solutions $\left\{u_{i}\right\}$ of (3.3) with $t=t_{i} \rightarrow t_{0} \in[0,1]$, and

$$
\max _{M^{n}} u_{i} \rightarrow \infty
$$

By Proposition 3.3, $\left\{u_{i}\right\}$ satisfies

$$
\max _{M^{n}}\left[\left|\nabla u_{i}\right|^{2}+\left|\nabla^{2} u_{i}\right|\right] \leq C,
$$

for some constant $C$ which is independent of $i$. In particular we see that

$$
u_{i} \rightarrow+\infty \text { uniformly. }
$$

Choose $x_{0} \in M^{n}$, and let

$$
w_{i}(x)=u_{i}(x)-u_{i}\left(x_{0}\right)
$$

Since

$$
w_{i}\left(x_{0}\right)=0
$$

by (4.6) $w_{i}$ satisfies the bounds

$$
\max _{M^{n}}\left[\left|w_{i}\right|+\left|\nabla w_{i}\right|^{2}+\left|\nabla^{2} w_{i}\right|\right] \leq C .
$$


Therefore, a subsequence of $\left\{w_{i}\right\}$ converges in $C^{1, \alpha}\left(M^{n}\right)$, any $\alpha \in(0,1)$, to $w \in$ $C^{1}\left(M^{n}\right)$. Moreover, since $w_{i}$ differs from $u_{i}$ by a constant, it is a solution of

$$
\begin{aligned}
& \operatorname{det}\left(A+\lambda e^{-2 u_{i}} g+\nabla^{2} w_{i}+d w_{i} \otimes d w_{i}-\frac{1}{2}\left|d w_{i}\right|^{2} g\right)^{1 / n} \\
& =t \mu e^{-2 u_{i}}+(1-t) f_{0}(x)
\end{aligned}
$$

with

$$
A+\lambda e^{-2 u_{i}} g+\nabla^{2} w_{i}+d w_{i} \otimes d w_{i}-\frac{1}{2}\left|d w_{i}\right|^{2} g>0
$$

Tracing this inequality (or, equivalently, applying the A-G-M inequality to (4.11)) and using the fact that $\sigma_{1}(A)=0$ we find

$$
n \lambda e^{-2 u_{i}}+\Delta w_{i}-\left(\frac{n-2}{2}\right)\left|\nabla w_{i}\right|^{2} \geq 0 .
$$

Therefore, by (4.7), in the limit $w=\lim _{i} w_{i}$ satisfies

$$
\Delta w \geq 0
$$

say, in an $H^{1}$-sense. It follows that $w$ is constant; in fact, by (4.9), $w \equiv 0$. Taking the limit in (4.12) we actually conclude $A \geq 0$. A little more precisely, let $X \in$ $\mathfrak{X}\left(M^{n}\right)$ be a smooth vector field on $M^{n}$; then by (4.12) we have

$$
\begin{aligned}
& \int\left[A_{k l} X^{k} X^{l}+\lambda e^{-2 u_{i}} g(X, X)+\left(\nabla_{k} \nabla_{l} w_{i}\right) X^{k} X^{l}\right. \\
& \left.+\left|d w_{i}(X)\right|^{2}-\frac{1}{2}\left|d w_{i}\right|^{2} g(X, X)\right]>0 .
\end{aligned}
$$

After integrating by parts we can write the Hessian term as

$$
\int\left(\nabla_{k} \nabla_{l} w_{i}\right) X^{k} X^{l}=-\int \nabla_{l} w_{i} \nabla_{k}\left(X^{k} X^{l}\right) \rightarrow 0, \quad i \rightarrow \infty
$$

since $\left\{w_{i}\right\}$ converges to zero in $C^{1}$. Letting $i \rightarrow \infty$ in (4.14) we find

$$
\int A_{k l} X^{k} X^{l} \geq 0, \quad \forall X \in \mathfrak{X}\left(M^{n}\right) .
$$

Therefore, $A \geq 0$.

Since $A$ is trace-free and $A \geq 0$, it follows that $A=0$, i.e., $g$ is Ricci-flat. However, this is a contradiction, so in fact (4.4) must hold.

Combining the results of Propositions 3.2, 3.3, 4.1, and 4.3, we have 
Proposition 4.4. Suppose $u \in C^{4, \alpha}$ is a solution of (3.3), with $t \in[0,1]$. If the scalar curvature of $g$ is negative; or if $g$ is scalar-flat but not Ricci-flat, then for any $k$ there is a constant $C=C(k)$ such that

$$
\|u\|_{C^{k}} \leq C .
$$

Proof. Under the assumptions of the proposition we have established that any solution $u \in C^{4, \alpha}$ of (3.3) satisfies

$$
\max _{M^{n}}\left[|u|+|\nabla u|+\left|\nabla^{2} u\right|\right] \leq C .
$$

It follows that $u$ is the solution of a uniformly elliptic, concave equation. By the results of Evans [3] and Krylov [9], we have the uniform estimate

$$
\|u\|_{C^{2, \alpha}} \leq C .
$$

Therefore, estimates of higher derivatives follow from classical elliptic regularity and the Schauder estimates.

Proposition 4.5. If the scalar curvature of $g$ is negative, or if $g$ is scalar-flat but not Ricci-flat, then $\mathfrak{S}$ is closed.

This concludes the proof of Theorem 1.2.

\section{The case of positive scalar curvature}

The proof of Proposition 4.3 can be adapted in order to prove a more general result:

Proposition 5.1. Suppose $u \in C^{2}$ is a solution of (3.3) with $t \in[0,1]$. If $\mathcal{W}_{n}([g])=$ $\emptyset$, then there is a constant $C=C(\mathrm{~g})$, independent of $t$, such that

$$
u \leq C \text {. }
$$

Proof. As in the proof of Proposition 4.3, suppose to the contrary that we have a sequence of solutions $\left\{u_{i}\right\}$ of (3.3) with $t=t_{i} \rightarrow t_{0} \in[0,1]$, and

$$
\max _{M^{n}} u_{i} \rightarrow \infty \text {. }
$$

By Proposition 3.3, $\left\{u_{i}\right\}$ satisfies

$$
\max _{M^{n}}\left[\left|\nabla u_{i}\right|^{2}+\left|\nabla^{2} u_{i}\right|\right] \leq C,
$$

for some constant $C$ which is independent of $i$. In particular we see that

$$
u_{i} \rightarrow+\infty \text { uniformly. }
$$


Choose $x_{0} \in M^{n}$, and let

$$
w_{i}(x)=u_{i}(x)-u_{i}\left(x_{0}\right)
$$

Since

$$
w_{i}\left(x_{0}\right)=0
$$

by (5.3) $w_{i}$ satisfies the bounds

$$
\max _{M^{n}}\left[\left|w_{i}\right|+\left|\nabla w_{i}\right|^{2}+\left|\nabla^{2} w_{i}\right|\right] \leq C .
$$

Therefore, a subsequence of $\left\{w_{i}\right\}$ converges in $C^{1, \alpha}\left(M^{n}\right)$, any $\alpha \in(0,1)$, to $w \in$ $C^{1}\left(M^{n}\right)$. Moreover, since $w_{i}$ differs from $u_{i}$ by a constant, it satisfies the equation

$$
\begin{aligned}
& \operatorname{det}\left(A+\lambda e^{-2 u_{i}} g+\nabla^{2} w_{i}+d w_{i} \otimes d w_{i}-\frac{1}{2}\left|d w_{i}\right|^{2} g\right)^{1 / n} \\
& =t \mu e^{-2 u_{i}}+(1-t) f_{0}(x)
\end{aligned}
$$

In particular, for each $i$ we have

$$
A+\lambda e^{-2 u_{i}} g+\nabla^{2} w_{i}+d w_{i} \otimes d w_{i}-\frac{1}{2}\left|d w_{i}\right|^{2} g>0 .
$$

However, it easily follows that $w \in \mathcal{W}_{n}([g])$, since $\lambda e^{-2 u_{i}} \rightarrow 0$ uniformly.

Corollary 5.2. If $\mathcal{W}_{n}([g])=\emptyset$ then $\mathfrak{S}$ is closed.

This immediately gives

Theorem 5.3. Let $\left(M^{n}, g\right)$ be a smooth, closed Riemannian manifold of dimension $n \geq 3$. Suppose $g$ is $\lambda$-admissible for some $\lambda>0$.

If $\mathcal{W}_{n}([g])=\emptyset$, then for each $0<\mu<\lambda$ there is a $\lambda$-admissible conformal metric $\widehat{g}=e^{-2 u} g$ satisfying

$$
\operatorname{det}(\widehat{A}+\lambda \widehat{g})^{1 / n}=\mu
$$

Moreover, the set of all $\lambda$-admissible solutions of (5.10) is compact in $C^{k}\left(M^{n}\right)$, for any $k \geq 0$.

\section{Remarks.}

(1) Recall from Proposition 2.3 that when $\mathcal{W}_{n}([g]) \neq \emptyset$, then $\lambda \leq \mu$. Therefore, if we seek a solution to (3.1) with $\lambda<\mu$ then $\mathcal{W}_{n}([g])=\emptyset$ is in fact a necessary condition. 
(2) The proof of Proposition 4.5 can be subsumed into the proof of Proposition 5.2. That is, if we assume $\sigma_{1}\left(A_{g}\right)<0$ then it is not difficult to see that $\mathcal{W}_{n}([g])=\emptyset$. However, the proof of Proposition 5.1 is quite indirect. In addition, when the scalar curvature is negative we have an effective bound for solutions in Corollary 4.2.

If the scalar curvature is positive, then one can verify $\mathcal{W}_{n}([g])=\emptyset$ by a topological criterion:

Proposition 5.4. Suppose $M^{n}$ is a smooth, closed manifold of dimension $n \geq 3$ whose first Betti number $b_{1}\left(M^{n}\right) \neq 0$. If $[\mathrm{g}]$ is a positive conformal class of metrics on $M^{n}$, then $\mathcal{W}_{n}([g])=\emptyset$.

Proof. We can assume without loss of generality that $g$ itself has positive scalar curvature.

Assume to the contrary that $w \in \mathcal{W}_{n}([g]) \neq \emptyset$, and let $\left\{w_{i}\right\}$ be the sequence of functions converging to $w$ as described in Definition 2.2. Denote $g_{i}=e^{-2 w_{i}} g$, and to simplify notation let $\mathrm{Ric}_{i}, A_{i}$, and $R_{i}$ denote respectively the Ricci tensor, Schouten tensor, and scalar curvature of $g_{i}$.

Since $b_{1} \neq 0$, for each $g_{i}$ there is a harmonic one-form $\alpha_{i} \in H^{1}\left(M^{n}, \mathbb{R}\right)$. By the Weitzenbock formula,

$$
\begin{aligned}
\frac{1}{2} \Delta_{i}\left|\alpha_{i}\right|_{g_{i}}^{2} & =\left|\nabla_{g_{i}} \alpha_{i}\right|_{g_{i}}^{2}+\operatorname{Ric}_{i}\left(\alpha_{i}, \alpha_{i}\right) \\
& =\left|\nabla_{g_{i}} \alpha_{i}\right|_{g_{i}}^{2}+(n-2) A_{i}\left(\alpha_{i}, \alpha_{i}\right)+\frac{1}{2(n-1)} R_{i}\left|\alpha_{i}\right|_{g_{i}}^{2} .
\end{aligned}
$$

By the properties of $\left\{w_{i}\right\}$, given any $\delta>0$, for $i$ sufficiently large we have

$$
\frac{1}{2} \Delta_{i}\left|\alpha_{i}\right|_{g_{i}}^{2} \geq\left|\nabla_{g_{i}} \alpha_{i}\right|_{g_{i}}^{2}+\left[\frac{1}{2(n-1)} R_{i}-\delta(n-2)\right]\left|\alpha_{i}\right|_{g_{i}}^{2}
$$

Writing $R_{i}$ in terms of $R=R(g)$,

$$
\frac{1}{2(n-1)} R_{i}=\frac{1}{2(n-1)} R e^{2 w_{i}}+\Delta_{g_{i}} w_{i}+\frac{(n-2)}{2}\left|d w_{i}\right|_{g_{i}}^{2} .
$$

Therefore,

$$
\begin{aligned}
\frac{1}{2} \Delta_{i}\left|\alpha_{i}\right|_{g_{i}}^{2} \geq & \left|\nabla_{g_{i}} \alpha_{i}\right|_{g_{i}}^{2}+\left[\frac{1}{2(n-1)} R e^{2 w_{i}}-\delta(n-2)\right]\left|\alpha_{i}\right|_{g_{i}}^{2} \\
& +\left[\Delta_{g_{i}} w_{i}+\frac{(n-2)}{2}\left|d w_{i}\right|_{g_{i}}^{2}\right]\left|\alpha_{i}\right|_{g_{i}}^{2} .
\end{aligned}
$$

If we fix $\delta=\delta(\min R, \min w)>0$ small enough, then for $i$ sufficiently large we have

$$
\frac{1}{2(n-1)} R e^{2 w_{i}}-\delta(n-2)>\delta
$$


and consequently

$$
\frac{1}{2} \Delta_{i}\left|\alpha_{i}\right|_{g_{i}}^{2} \geq\left|\nabla_{g_{i}} \alpha_{i}\right|_{g_{i}}^{2}+\left[\Delta_{g_{i}} w_{i}+\frac{(n-2)}{2}\left|d w_{i}\right|_{g_{i}}^{2}+\delta\right]\left|\alpha_{i}\right|_{g_{i}}^{2} .
$$

Next, we multiply both sides of this inequality by $e^{\theta w_{i}}$, where $\theta=\theta(n)$ will be specified momentarily, and integrate by parts to get

$$
\begin{aligned}
0> & \int\left\{-\frac{1}{2} \Delta_{i}\left|\alpha_{i}\right|_{g_{i}}^{2}+\left|\nabla_{g_{i}} \alpha_{i}\right|_{g_{i}}^{2}+\left[\Delta_{g_{i}} w_{i}+\frac{(n-2)}{2}\left|d w_{i}\right|_{g_{i}}^{2}+\delta\right]\left|\alpha_{i}\right|_{g_{i}}^{2}\right\} e^{\theta w_{i}} d V_{g_{i}} \\
= & \int\left\{\left|\nabla_{g_{i}} \alpha_{i}\right|_{g_{i}}^{2}+\left(\frac{\theta}{2}-1\right)\left\langle\nabla_{g_{i}} w_{i}, \nabla_{g_{i}}\left|\alpha_{i}\right|_{g_{i}}^{2}\right\rangle_{g_{i}}\right. \\
& \left.+\left(\frac{n-2}{2}-\theta\right)\left|d w_{i}\right|_{g_{i}}^{2}\left|\alpha_{i}\right|_{g_{i}}^{2}+\delta\left|\alpha_{i}\right|_{g_{i}}^{2}\right\} e^{\theta w_{i}} d V_{g_{i}} .
\end{aligned}
$$

We need to consider two cases. First, suppose the dimension $n \geq 4$. Taking $\theta=0$ in (5.15), using Kato's inequality

$$
\left|\nabla_{g_{i}} \alpha_{i}\right|_{g_{i}}^{2} \geq\left.\left|\nabla_{g_{i}}\right| \alpha_{g_{i}}\right|_{g_{i}} ^{2},
$$

and completing the square, we find

$$
\begin{aligned}
0> & \int\left\{\left|\nabla_{g_{i}}\right| \alpha_{i}||_{g_{i}}^{2}-2\left\langle\nabla_{g_{i}} w_{i}, \nabla_{g_{i}}\left|\alpha_{i}\right|_{g_{i}}\right\rangle_{g_{i}}\left|\alpha_{i}\right|_{g_{i}}\right. \\
& \left.+\frac{(n-2)}{2}\left|d w_{i}\right|_{g_{i}}^{2}\left|\alpha_{i}\right|_{g_{i}}^{2}+\delta\left|\alpha_{i}\right|_{g_{i}}^{2}\right\} d V_{g_{i}} \\
\geq & \int\left\{\frac{(n-4)}{2}\left|d w_{i}\right|_{g_{i}}^{2}\left|\alpha_{i}\right|_{g_{i}}^{2}+\delta\left|\alpha_{i}\right|_{g_{i}}^{2}\right\} d V_{g_{i}},
\end{aligned}
$$

a contradiction.

When $n=3$ we need a refined version of (5.16):

$$
\left|\nabla_{g_{i}} \alpha_{i}\right|_{g_{i}}^{2} \geq\left.\frac{n}{n-1}\left|\nabla_{g_{i}}\right| \alpha_{i}\right|_{g_{i}} ^{2}
$$

which follows from the Cauchy-Schwarz inequality (see [1] for a more general perspective). Taking $\theta=-1$ in (5.15) and using (5.18), we get

$$
\begin{aligned}
0> & \int\left\{\frac{3}{2}\left|\nabla_{g_{i}}\right| \alpha_{i}||_{g_{i}}^{2}-3\left\langle\nabla_{g_{i}} w_{i}, \nabla_{g_{i}}\left|\alpha_{i}\right|_{g_{i}}\right\rangle_{g_{i}}\left|\alpha_{i}\right|_{g_{i}}\right. \\
& \left.+\frac{3}{2}\left|d w_{i}\right|_{g_{i}}^{2}\left|\alpha_{i}\right|_{g_{i}}^{2}+\delta\left|\alpha_{i}\right|_{g_{i}}^{2}\right\} e^{-w_{i}} d V_{g_{i}} \\
\geq & \int \delta\left|\alpha_{i}\right|_{g_{i}}^{2} e^{-w_{i}} d V_{g_{i}}
\end{aligned}
$$

which is once again a contradiction. Therefore, we must have $\mathcal{W}_{n}([g])=\emptyset$. 


\subsection{The proof of Theorem 1.4}

To prove Theorem 1.4, suppose $g$ is a metric of positive scalar curvature on $M^{n}$, where $b_{1}\left(M^{n}\right) \neq 0$. Given numbers $0<\mu<\lambda$, we can (if necessary) rescale $g$ to make it $\lambda$-admissible. Then Theorem 5.3 and Proposition 5.4 imply the existence of a $\lambda$-admissible solution $\widehat{g}=e^{-2 u} g$ of

$$
\operatorname{det}(\widehat{A}+\lambda \widehat{g})^{1 / n}=\mu,
$$

as claimed.

\section{Non-uniqueness}

Because equation (1.3) is invariant under the action of the conformal group, solutions are in general not unique. In this section we show that uniqueness also fails in for negative conformal classes. Our construction is an adaptation of Schoen's construction of the Delunay metrics on $S^{n-1} \times S^{1}$ [10], and it is rather surprising that for our equation one can replace the sphere factor with a hyperbolic factor.

To begin, let $\left(\mathbb{H}^{n-1}, d s^{2}\right)$ denote a compact hyperbolic manifold of dimension $n-1$, where $n \geq 3$. Assume the Ricci curvature of $d s^{2}$ is normalized to be $-(n-2)$. Let $\left(M^{n}, g\right)$ be the product manifold,

$$
M^{n}=\mathbb{H}^{n-1} \times \mathbb{R}, \quad g=d s^{2}+d t^{2} .
$$

Then the Schouten tensor of $g$ is given by

$$
A=\left(\begin{array}{cccc}
-1 / 2 & & & \\
& \ddots & & \\
& & -1 / 2 & \\
& & & 1 / 2
\end{array}\right) .
$$

Note that $g$ is $\lambda$-admissible for any $\lambda>1 / 2$.

Suppose $\widehat{g}=e^{-2 u} g$ is a solution of (1.4):

$$
\operatorname{det}\left(A+\lambda e^{-2 u} g+\nabla^{2} u+d u \otimes d u-\frac{1}{2}|d u|^{2} g\right)^{1 / n}=\mu e^{-2 u} .
$$

Let $w=e^{u}$, then $w$ is a solution of

$$
\operatorname{det}\left(w^{2} A+\lambda g+w \nabla^{2} w-\frac{1}{2}|d w|^{2} g\right)^{1 / n}=\mu .
$$

Note that $w_{0} \equiv 1$ is a solution of (6.3) with $\lambda=1$ and $\mu=(1 / 2) 3^{1 / n}$. Our goal is to find other solutions $w=w(t)$ which are periodic, and therefore descend to give 
solutions on $\mathbb{H}^{n-1} \times S^{1}$. To this end, suppose $w=w(t)$; then (6.3) is equivalent to

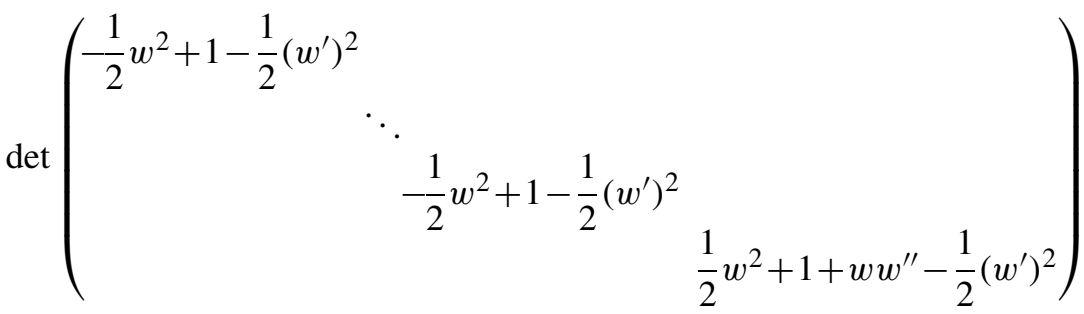

$$
\begin{aligned}
& =3\left(\frac{1}{2}\right)^{n},
\end{aligned}
$$

which gives the ODE

$$
\left\{-\frac{1}{2} w^{2}+1-\frac{1}{2}\left(w^{\prime}\right)^{2}\right\}^{n-1}\left\{\frac{1}{2} w^{2}+1+w w^{\prime \prime}-\frac{1}{2}\left(w^{\prime}\right)^{2}\right\}=3\left(\frac{1}{2}\right)^{n} .
$$

Interestingly, there is a conserved quantity associated to the flow (6.5). For periodic solutions of the $k$-Yamabe problem on $S^{n-1} \times S^{1}$ a conserved quantity was written down in [12] by using the variational structure of the PDE and Noether's Theorem. Here, however, there is no obvious variational structure, and our quantity was discovered more or less by trial and error:

Lemma 6.1. Let $x=x(t)>0$, and define

$$
D(t)=x^{-n}\left\{-3\left(\frac{1}{2}\right)^{n}+\left[1-\frac{1}{2} x^{2}-\frac{1}{2}\left(x^{\prime}\right)^{2}\right]^{n}\right\} .
$$

Then $D(\cdot)$ is constant if and only if $w=x(t)$ is a solution of $(6.5)$.

Using this lemma we can reduce the second order equation to first order one and study its phase portrait. As we observed above, $(1,0)$ is a stationary point of the phase flow. Nearby, there are two families of orbits. Points on the $x$-axis $\left(x_{0}, 0\right)$ with $x_{0}<1$ lie on orbits which approach the $x^{\prime}$-axis, and therefore do not correspond to conformal metrics. Points with $x_{0}>1$ (but close to 1) lie on closed orbits with $x(t)>0$ for all time, and therefore give non-trivial solutions of (6.4) on $\mathbb{H}^{n-1} \times S^{1}$; see Figure 6.1 below.

\section{An application}

In this section we prove

Theorem 7.1. If $g$ has negative scalar curvature, or if $g$ is scalar-flat but not Ricciflat, then there is a $C^{1,1}$-conformal metric $\widehat{g}=e^{-2 u} g$ with

$$
\rho_{\min }[\widehat{A}]=-1 / 2 \text { a.e. }
$$




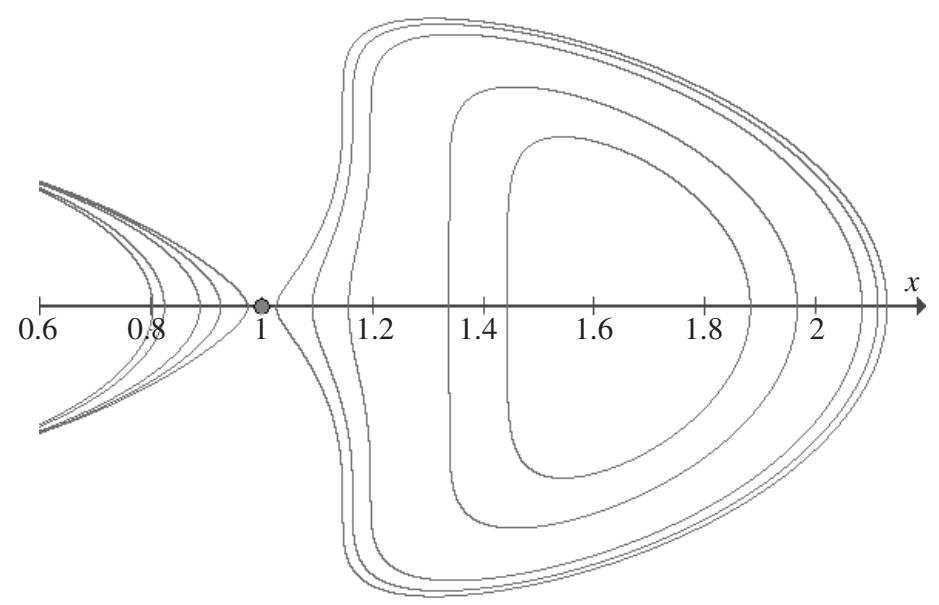

Figure 6.1. Some closed orbits for $n=3$.

Proof. Suppose $g$ satisfies the hypotheses of the Theorem. Let $\lambda=1 / 2$, and $1 / 2>$ $\mu_{j} \searrow 0$ be a sequence of positive numbers converging to zero. By Theorem 1.2, for each $j$ there is a $1 / 2$-admissible solution $g_{j}=e^{-2 u_{j}} g$ of

$$
\operatorname{det}\left(A+\frac{1}{2} e^{-2 u_{j}} g+\nabla^{2} u_{j}+d u_{j} \otimes d u_{j}-\frac{1}{2}\left|d u_{j}\right|^{2} g\right)^{1 / n}=\mu_{j} e^{-2 u_{j}}
$$

By Proposition 3.2, there is a constant $C=C(g)>0$ such that

$$
\begin{aligned}
u_{j} & \geq \frac{1}{2} \log \left(\frac{1}{2}-\mu_{j}\right)-C \\
& \geq-C .
\end{aligned}
$$

Therefore, by Corollary 8.3 in the Appendix we have bounds on the gradient and Hessian as well:

$$
\max \left[\left|\nabla^{2} u_{j}\right|+\left|\nabla u_{j}\right|^{2}\right] \leq C
$$

If $g$ has negative scalar curvature, then by Proposition $4.1\left\{u_{j}\right\}$ has a uniform upper bound, hence

$$
\max \left[\left|\nabla^{2} u_{j}\right|+\left|\nabla u_{j}\right|^{2}+\left|u_{j}\right|\right] \leq C
$$

On the other hand, if $g$ is scalar-flat but not Ricci-flat, we can argue exactly as in the proof of Proposition 4.3 to show that $u_{j}$ is bounded above in this case as well. 
Therefore, there is a subsequence (also denoted $\left\{u_{j}\right\}$ ), and $u \in C^{1,1}\left(M^{n}\right)$, such that $u_{j} \rightarrow u$ in $C^{1, \alpha}$, for any $\alpha \in(0,1)$. Moreover, by (7.2) the limit $u$ satisfies

$$
\operatorname{det}\left(A+\frac{1}{2} e^{-2 u} g+\nabla^{2} u+d u \otimes d u-\frac{1}{2}|d u|^{2} g\right)^{1 / n}=0 \quad \text { a.e. }
$$

\section{Appendix: $C^{2}$-estimates}

In this Appendix we prove local estimates for solutions of

$$
\operatorname{det}\left(A+\lambda e^{-2 u} g+\nabla^{2} u+d u \otimes d u-\frac{1}{2}|d u|^{2} g\right)^{1 / n}=t \mu e^{-2 u}+f(x),
$$

where $f$ is a smooth positive function and $t \in[0,1]$. Note that (3.3) is an example of this equation, as is (1.4).

Our proof is based very closely on the work of S. Chen, who established local estimates for a fairly general class of fully nonlinear second order equations [2] though none include the additional exponential nonlinearity as in (8.1). Therefore, we need to make some minor adaptations of her argument. The proofs we give also apply to the generalizations of (8.1) mentioned in the Introduction; for example, by replacing the determinant with another elementary symmetric function or the Schouten tensor with the Ricci tensor.

Our main estimate is the following:

Theorem 8.1. Suppose $u \in C^{4}$ is a solution of (8.1) in $B(O, r) \subset M^{n}$, and that $\widehat{g}=e^{-2 u} g$ is $\lambda$-admissible. Then there is a constant

$$
C=C\left(r, n,\|g\|_{C^{3}},\|f\|_{C^{2}}\right)
$$

such that

$$
\max _{B(O, r / 2)}\left[|\Delta u|+|\nabla u|^{2}\right] \leq C\left(1+|\lambda-t \mu| \max _{B(O, r)} e^{-2 u}\right) .
$$

Proof. We first note that, by the A-G-M inequality,

$$
\begin{aligned}
f(x)+t \mu e^{-2 u} & \leq \frac{1}{n} \sigma_{1}\left(A+\lambda e^{-2 u} g+\nabla^{2} u+d u \otimes d u-\frac{1}{2}|d u|^{2}\right) \\
& =\frac{1}{n} \sigma_{1}(A)+\frac{1}{n} \Delta u-\frac{(n-2)}{2 n}|\nabla u|^{2}
\end{aligned}
$$

hence

$$
\Delta u \geq \frac{(n-2)}{2}|\nabla u|^{2}+n(t \mu-\lambda) e^{-2 u}-C(g, f) .
$$


Given this lower bound, to prove (8.26) it suffices to prove

$$
\max _{B(O, r / 2)}\left[\Delta u+|\nabla u|^{2}\right] \leq C\left(1+|\lambda-t \mu| \max _{B(O, r)} e^{-2 u}\right) .
$$

Moreover, by a simple scaling argument we can recover the result if we first prove the estimate on a ball of radius 1 .

To this end, let $\eta \in C^{\infty}$ be a smooth cut-off function with $0 \leq \eta \leq 1, \eta \equiv 1$ in $B(O, 1 / 2)$, supp $\eta \subset B(O, 3 / 4)$, and $|\nabla \eta|^{2} / \eta \leq C$. Denote

$$
H=\left(\Delta u+|\nabla u|^{2}\right) \eta \text {. }
$$

Then

$$
\nabla_{j} H=\eta \nabla_{j}\left(\Delta u+|\nabla u|^{2}\right)+\left(\Delta u+|\nabla u|^{2}\right) \nabla_{j} \eta .
$$

At a critical point of $H$,

$$
\nabla_{j}\left(\Delta u+|\nabla u|^{2}\right)=-\left(\Delta u+|\nabla u|^{2}\right) \frac{\nabla_{j} \eta}{\eta} .
$$

Differentiating again,

$$
\begin{aligned}
\nabla_{i} \nabla_{j} H= & \eta \nabla_{i} \nabla_{j}\left(\Delta u+|\nabla u|^{2}\right)+\nabla_{i}\left(\Delta u+|\nabla u|^{2}\right) \nabla_{j} \eta+\nabla_{i}(\Delta u \\
& \left.+|\nabla u|^{2}\right) \nabla_{j} \eta+\left(\Delta u+|\nabla u|^{2}\right) \nabla_{i} \nabla_{j} \eta .
\end{aligned}
$$

At a point where $H$ attains its maximum, using (8.7) this becomes

$$
\begin{aligned}
0 \geq & \eta^{-1} \nabla_{i} \nabla_{j} H=\nabla_{i} \nabla_{j}\left(\Delta u+|\nabla u|^{2}\right) \\
& +\left(\frac{\nabla_{i} \nabla_{j} \eta}{\eta}-2 \frac{\nabla_{i} \eta \nabla_{j} \eta}{\eta^{2}}\right)\left(\Delta u+|\nabla u|^{2}\right) .
\end{aligned}
$$

Consider the highest order terms in (8.8):

$$
\begin{aligned}
\nabla_{i} \nabla_{j}\left(\Delta u+|\nabla u|^{2}\right) & =\nabla_{i} \nabla_{j}(\Delta u)+\nabla_{i} \nabla_{j}|\nabla u|^{2} \\
& =I+I I .
\end{aligned}
$$

We will calculate $I$ and $I I$ separately.

For $I$, after commuting derivatives we find

$$
I=\nabla_{i} \nabla_{j}(\Delta u)=\Delta \nabla_{i} \nabla_{j} u+O\left(\left|\nabla^{2} u\right|+|\nabla u|\right),
$$

where $O(x)$ will denote a quantity which can be bounded by $C|x|$, with $C=$ $C(n, g, f)$. Denote

$$
W_{i j}=A_{i j}+\lambda e^{-2 u} g_{i j}+\nabla_{i} \nabla_{j} u+\nabla_{i} u \nabla_{j} u-\frac{1}{2}|\nabla u|^{2} g_{i j} .
$$


Then the main term in $(8.10)$ is

$$
\begin{aligned}
\Delta \nabla_{i} \nabla_{j} u & =\Delta\left[W_{i j}-A_{i j}-\lambda e^{-2 u} g_{i j}-\nabla_{i} u \nabla_{j} u+\frac{1}{2}|\nabla u|^{2} g_{i j}\right] \\
& =\Delta W_{i j}-\lambda \Delta\left(e^{-2 u}\right) g_{i j}-\Delta\left(\nabla_{i} u \nabla_{j} u\right)+\frac{1}{2}\left(\Delta|\nabla u|^{2}\right) g_{i j}+O(1) .
\end{aligned}
$$

Applying the Leibniz rule and commuting derivatives again, we find

$$
\begin{aligned}
I= & \Delta W_{i j}-\lambda \Delta\left(e^{-2 u}\right) g_{i j}-\nabla_{i}(\Delta u) \nabla_{j} u-\nabla_{j}(\Delta u) \nabla_{i} u+\langle\nabla(\Delta u), \nabla u\rangle g_{i j} \\
& -2 \nabla_{i} \nabla_{k} u \nabla_{j} \nabla_{k} u+\left|\nabla^{2} u\right|^{2} g_{i j}+O\left(\left|\nabla^{2} u\right|+|\nabla u|^{2}+1\right) .
\end{aligned}
$$

Next, we estimate $I I$ :

$$
\begin{aligned}
I I & =\nabla_{i} \nabla_{j}|\nabla u|^{2} \\
& =2 \nabla_{i} \nabla_{k} u \nabla_{j} \nabla_{k} u+2 \nabla_{i} \nabla_{j} \nabla_{k} u \nabla_{k} u .
\end{aligned}
$$

For the second term, we commute derivatives and use (8.11) to write

$$
\begin{aligned}
2 \nabla_{i} \nabla_{j} \nabla_{k} u \nabla_{k} u= & 2 \nabla_{k}\left(\nabla_{i} \nabla_{j} u\right) \nabla_{k} u+O\left(|\nabla u|^{2}\right) \\
= & 2 \nabla_{k}\left[W_{i j}-A_{i j}-\lambda e^{-2 u} g_{i j}-\nabla_{i} u \nabla_{j} u+\frac{1}{2}|\nabla u|^{2} g_{i j}\right] \nabla_{k} u \\
& +O\left(|\nabla u|^{2}\right) \\
= & 2 \nabla_{k} W_{i j} \nabla_{k} u-2 \lambda\left\langle\nabla\left(e^{-2 u}\right), \nabla u\right\rangle g_{i j}-2 \nabla_{i} \nabla_{k} u \nabla_{j} u \nabla_{k} u \\
& -2 \nabla_{j} \nabla_{k} u \nabla_{i} u \nabla_{k} u+\left\langle\nabla|\nabla u|^{2}, \nabla u\right\rangle g_{i j}+O\left(|\nabla u|^{2}+1\right) \\
= & 2 \nabla_{k} W_{i j} \nabla_{k} u-2 \lambda\left\langle\nabla\left(e^{-2 u}\right), \nabla u\right\rangle g_{i j}-\nabla_{i}|\nabla u|^{2} \nabla_{j} u-\nabla_{j}|\nabla u|^{2} \nabla_{i} u \\
& +\left\langle\nabla|\nabla u|^{2}, \nabla u\right\rangle g_{i j}+O\left(|\nabla u|^{2}+1\right) .
\end{aligned}
$$

Hence,

$$
\begin{aligned}
I I= & 2 \nabla_{k} W_{i j} \nabla_{k} u-2 \lambda\left\langle\nabla\left(e^{-2 u}\right), \nabla u\right\rangle g_{i j}+2 \nabla_{i} \nabla_{k} u \nabla_{j} \nabla_{k} u \\
& -\nabla_{i}|\nabla u|^{2} \nabla_{j} u-\nabla_{j}|\nabla u|^{2} \nabla_{i} u \\
& +\left\langle\nabla|\nabla u|^{2}, \nabla u\right\rangle g_{i j}+O\left(|\nabla u|^{2}+1\right) .
\end{aligned}
$$

Adding (8.12) and (8.13), we have

$$
\begin{aligned}
I+I I= & \Delta W_{i j}+2 \nabla_{k} W_{i j} \nabla_{k} u-\lambda\left[\Delta\left(e^{-2 u}\right)+2\left\langle\nabla\left(e^{-2 u}\right), \nabla u\right\rangle\right] g_{i j} \\
& -\nabla_{i}\left(\Delta u+|\nabla u|^{2}\right) \nabla_{j} u-\nabla_{i}\left(\Delta u+|\nabla u|^{2}\right) \nabla_{j} u \\
& +\left\langle\nabla\left(\Delta u+|\nabla u|^{2}\right), \nabla u\right\rangle g_{i j}+\left|\nabla^{2} u\right|^{2} g_{i j}+O\left(\left|\nabla^{2} u\right|+|\nabla u|^{2}+1\right) \\
= & \Delta W_{i j}+2 \nabla_{k} W_{i j} \nabla_{k} u-\lambda\left[\Delta\left(e^{-2 u}\right)+2\left\langle\nabla\left(e^{-2 u}\right), \nabla u\right\rangle\right] g_{i j} \\
& +\left(\Delta u+|\nabla u|^{2}\right)\left[\nabla_{i} u \frac{\nabla_{j} \eta}{\eta}+\nabla_{j} u \frac{\nabla_{i} \eta}{\eta}-\left\langle\nabla u, \frac{\nabla \eta}{\eta}\right\rangle g_{i j}\right] \\
& +\left|\nabla^{2} u\right|^{2} g_{i j}+O\left(\left|\nabla^{2} u\right|+|\nabla u|^{2}+1\right) .
\end{aligned}
$$


As in the proof of Proposition 3.1, let

$$
\begin{aligned}
F(W) & =\operatorname{det}(W)^{1 / n}, \\
F^{i j} & =\frac{\partial F}{\partial W_{i j}} .
\end{aligned}
$$

At the maximum point of $H$,

$$
0 \geq F^{i j} \nabla_{i} \nabla_{j} H .
$$

Therefore, substituting (8.14) into (8.9) and (8.8) and using (8.15), we have

$$
\begin{aligned}
0 \geq & \eta^{-1} F^{i j} \nabla_{i} \nabla_{j} H=F^{i j}\left\{\Delta W_{i j}+2 \nabla_{k} W_{i j} \nabla_{k} u\right. \\
& -\lambda\left[\Delta\left(e^{-2 u}\right)+2\left\langle\nabla\left(e^{-2 u}\right), \nabla u\right\rangle\right] g_{i j} \\
& +\left(\Delta u+|\nabla u|^{2}\right)\left[\nabla_{i} u \frac{\nabla_{j} \eta}{\eta}+\nabla_{j} u \frac{\nabla_{i} \eta}{\eta}-\left\langle\nabla u, \frac{\nabla \eta}{\eta}\right\rangle g_{i j}\right. \\
& \left.+\frac{\nabla_{i} \nabla_{j} \eta}{\eta}-2 \frac{\nabla_{i} \eta \nabla_{j} \eta}{\eta^{2}}\right] \\
& \left.+\left|\nabla^{2} u\right|^{2} g_{i j}+O\left(\left|\nabla^{2} u\right|+|\nabla u|^{2}+1\right)\right\} .
\end{aligned}
$$

The dominant term in (8.16) is the Hessian term, which we estimate (along with the corresponding lower order term) as

$$
\begin{aligned}
\left|\nabla^{2} u\right|^{2}+O\left(\left|\nabla^{2} u\right|\right) & \geq \frac{1}{2}\left|\nabla^{2} u\right|^{2}+O(1) \\
& \geq \frac{1}{2 n}(\Delta u)^{2}+O(1) .
\end{aligned}
$$

Now,

$$
\left(\Delta u+|\nabla u|^{2}\right)^{2} \leq 2(\Delta u)^{2}+2|\nabla u|^{4},
$$

while (8.4) implies

$$
|\nabla u|^{4} \leq c_{n}(\Delta u)^{2}+C(t \mu-\lambda)^{2} e^{-4 u}+C,
$$

where $c_{n}$ is a positive constant depending only on $n$. Therefore,

$$
\left(\Delta u+|\nabla u|^{2}\right)^{2} \leq c_{n}^{\prime}(\Delta u)^{2}+C(t \mu-\lambda)^{2} e^{-4 u}+C .
$$

Combining (8.17) and (8.18) we find

$$
\left|\nabla^{2} u\right|^{2}+O\left(\left|\nabla^{2} u\right|\right) \geq \epsilon_{n}\left(\Delta u+|\nabla u|^{2}\right)^{2}-C(t \mu-\lambda)^{2} e^{-4 u}+O(1),
$$

for some $\epsilon_{n}>0$ which only depends on the dimension. 
Next, we estimate those terms in (8.16) involving derivatives of $W$. First, by the concavity of $F$,

$$
\begin{aligned}
F^{i j} \Delta W_{i j} & \geq \Delta F(W) \\
& =\Delta\left(t \mu e^{-2 u}+f\right) \\
& =t \mu \Delta\left(e^{-2 u}\right)+O(1) .
\end{aligned}
$$

Also, by differentiating equation (8.1),

$$
\begin{aligned}
2 F^{i j} \nabla_{k} W_{i j} \nabla_{k} u & =2 \nabla_{k} F(W) \nabla_{k} u \\
& =2 \nabla_{k}\left(t \mu e^{-2 u}+f\right) \nabla_{k} u \\
& =2 t \mu\left\langle\nabla\left(e^{-2 u}\right), \nabla u\right\rangle+O(|\nabla u|+1) .
\end{aligned}
$$

Substituting these along with (8.19) into (8.16) we get

$$
\begin{aligned}
0 \geq & \left(t \mu-\lambda F^{k k}\right)\left[\Delta\left(e^{-2 u}\right)+2\left\langle\nabla\left(e^{-2 u}\right), \nabla u\right\rangle\right] \\
& +F^{i j}\left\{\epsilon_{n}\left(\Delta u+|\nabla u|^{2}\right)^{2} g_{i j}-C(t \mu-\lambda)^{2} e^{-4 u} g_{i j}\right. \\
& +\left(\Delta u+|\nabla u|^{2}\right)\left[\nabla_{i} u \frac{\nabla_{j} \eta}{\eta}+\nabla_{j} u \frac{\nabla_{i} \eta}{\eta}-\left\langle\nabla u, \frac{\nabla \eta}{\eta}\right\rangle g_{i j}\right. \\
& \left.+\frac{\nabla_{i} \nabla_{j} \eta}{\eta}-2 \frac{\nabla_{i} \eta \nabla_{j} \eta}{\eta^{2}}\right] \\
& \left.+O\left(|\nabla u|^{2}+1\right)\right\}
\end{aligned}
$$

Since $F^{k k} \geq 1$,

$$
\left(t \mu-\lambda F^{k k}\right) \leq(t \mu-\lambda) .
$$

Also,

$$
\Delta\left(e^{-2 u}\right)+2\left\langle\nabla\left(e^{-2 u}\right), \nabla u\right\rangle=-2 \Delta u e^{-2 u} .
$$

We may assume $\Delta u \geq 0$; otherwise by (8.4) we would be done. Therefore,

$$
\begin{aligned}
\left(t \mu-\lambda F^{k k}\right)\left[\Delta\left(e^{-2 u}\right)+2\left\langle\nabla\left(e^{-2 u}\right), \nabla u\right\rangle\right] & \geq-2(t \mu-\lambda) \Delta u e^{-2 u} \\
& \geq-2|t \mu-\lambda| e^{-2 u}\left(\Delta u+|\nabla u|^{2}\right) .
\end{aligned}
$$


Substituting this into (8.20) gives

$$
\begin{aligned}
0 \geq & -2|t \mu-\lambda| e^{-2 u}\left(\Delta u+|\nabla u|^{2}\right) \\
& +F^{i j}\left\{\epsilon_{n}\left(\Delta u+|\nabla u|^{2}\right)^{2} g_{i j}-C(t \mu-\lambda)^{2} e^{-4 u} g_{i j}\right. \\
& +\left(\Delta u+|\nabla u|^{2}\right)\left[\nabla_{i} u \frac{\nabla_{j} \eta}{\eta}+\nabla_{j} u \frac{\nabla_{i} \eta}{\eta}-\left\langle\nabla u, \frac{\nabla \eta}{\eta}\right\rangle g_{i j}\right. \\
& \left.+\frac{\nabla_{i} \nabla_{j} \eta}{\eta}-2 \frac{\nabla_{i} \eta \nabla_{j} \eta}{\eta^{2}}\right] \\
& \left.+O\left(|\nabla u|^{2}+1\right)\right\} \\
\geq & -2|t \mu-\lambda| e^{-2 u}\left(\Delta u+|\nabla u|^{2}\right) \\
& +F^{i j}\left\{\epsilon_{n}\left(\Delta u+|\nabla u|^{2}\right)^{2} g_{i j}-C_{1}(t \mu-\lambda)^{2} e^{-4 u} g_{i j}\right. \\
& -C_{2}\left(\Delta u+|\nabla u|^{2}\right)\left[|\nabla u| \frac{|\nabla \eta|}{\eta}+\frac{\left|\nabla^{2} \eta\right|}{\eta}+\frac{|\nabla \eta|^{2}}{\eta^{2}}\right] \\
& \left.+O\left(|\nabla u|^{2}+1\right)\right\} .
\end{aligned}
$$

Multiplying this inequality by $\eta^{2}$ and estimating the lower order terms in the obvious manner, we end up with

$$
\begin{aligned}
0 \geq & -2 \eta H|t \mu-\lambda| e^{-2 u} \\
& +F^{i j}\left\{\epsilon_{n} H^{2} g_{i j}-C_{1} \eta^{2}(t \mu-\lambda)^{2} e^{-4 u} g_{i j}\right. \\
& -C_{2} H\left[|\nabla u||\nabla \eta|+\left|\nabla^{2} \eta\right|+\frac{|\nabla \eta|^{2}}{\eta}\right] \\
& \left.+O\left(\eta^{2}|\nabla u|^{2}+1\right)\right\} \\
\geq & -2 \eta H|\mu-\lambda| e^{-2 u} \\
& +F^{i j}\left\{\frac{1}{2} \epsilon_{n} H^{2} g_{i j}-C \eta^{2}(t \mu-\lambda)^{2} e^{-4 u} g_{i j}+O(1)\right\} .
\end{aligned}
$$


If the term inside the braces is negative, then we are obviously done. Otherwise, using the inequality $F^{k k} \geq 1$ again we have

$$
0 \geq-2 \eta H|t \mu-\lambda| e^{-2 u}+\frac{1}{2} \epsilon_{n} H^{2} g_{i j}-C \eta^{2}(t \mu-\lambda)^{2} e^{-4 u} g_{i j}+O(1),
$$

which clearly implies (8.5).

We note two Corollaries of Theorem 8.1:

Corollary 8.2. Suppose $u \in C^{4}$ is a solution of (8.1) in $B(O, r) \subset M^{n}$, and that $\widehat{g}=e^{-2 u} g$ is $\lambda$-admissible. Then there is a constant

$$
C=C\left(\lambda, \mu, r, n,\|g\|_{C^{3}},\|f\|_{C^{2}}\right)
$$

such that

$$
\max _{B(O, r / 2)}\left[\left|\nabla^{2} u\right|+|\nabla u|^{2}\right] \leq C\left(1+\max _{B(O, r)} e^{-2 u}\right)
$$

Proof. Since $\widehat{g}$ is $\lambda$-admissible it follows that

$$
0<\sigma_{2}\left(A+\lambda e^{-2 u}+\nabla^{2} u+d u \otimes d u-\frac{1}{2}|d u|^{2} g\right)
$$

Expanding the expression for $\sigma_{2}$ one quickly finds

$$
\left|\nabla^{2} u\right|^{2} \leq C\left[(\Delta u)^{2}+|\nabla u|^{4}+\lambda^{2} e^{-4 u}+1\right]
$$

The result now follows from (8.26).

Corollary 8.3. Suppose $\widehat{g}=e^{-2 u} g$ is a $\lambda$-admissible solution of (8.1), where $u \in$ $C^{4}\left(M^{n}\right)$. Then there is a constant

$$
C=C\left(r, n,\|g\|_{C^{3}},\|f\|_{C^{2}}\right)
$$

such that

$$
\max _{M^{n}} \sigma_{1}\left(A+\nabla^{2} u+d u \otimes d u-\frac{1}{2}|d u|^{2} g\right) \leq C\left(1+|\lambda-t \mu| \max _{M^{n}} e^{-2 u}\right) .
$$




\section{References}

[1] T. P. BRAnson, Kato constants in Riemannian geometry, Math. Res. Lett. 7 (2000), 245261.

[2] S. S. CHEN, Local estimates for some fully nonlinear elliptic equations, Int. Math. Res. Not. 55 (2005), 3403-3425.

[3] L. C. Evans, Classical solutions of fully nonlinear, convex, second-order elliptic equations, Comm. Pure Appl. Math. 35 (1982), 333-363.

[4] M. J. GurSKy and J. A. Viaclovsky, A new variational characterization of threedimensional space forms, Invent. Math. 145 (2001) 251-278.

[5] M. J. GURSKY and J. A. VIAClOVSKY, A fully nonlinear equation on four-manifolds with positive scalar curvature, J. Differential Geom. 63 (2003), 131-154.

[6] M. J. GURSKY and J. A. VIACLOVSKY, Fully nonlinear equations on Riemannian manifolds with negative curvature, Indiana Univ. Math. J. 52 (2003), 399-419.

[7] P. GUAN and G. WANG, Geometric inequalities on locally conformally flat manifolds, Duke Math. J. 124 (2004), 177-212.

[8] P. GUAN and G. WANG, Conformal deformations of the smallest eigenvalue of the Ricci tensor, Amer. J. Math. 129 (2007), 499-526.

[9] N. V. KRYLOV, Boundedly inhomogeneous elliptic and parabolic equations in a domain, Izv. Akad. Nauk SSSR Ser. Mat. 47 (1983), 75-108.

[10] R. M. SCHOEN, Variational theory for the total scalar curvature functional for Riemannian metrics and related topics, Topics in calculus of variations, (Montecatini Terme, 1987), Lecture Notes in Math., Vol. 1365, Springer, Berlin, 1989, 120-154.

[11] W.-M. Sheng, N. S. TRUdinger and X.-J. WANG, The Yamabe problem for higher order curvatures, J. Differential Geom. 77 (2007), 515-553.

[12] J. A. ViaClovSKY, Conformal geometry, contact geometry, and the calculus of variations, Duke Math. J. 101 (2000), 283-316.

[13] J. A. ViAClOVSKY, Conformal geometry and differential equations, to appear in: "Inspired by S. S. Chern: A Memorial Volume in Honor of a Great Mathematician”, P. Griffiths (ed.), Nankai Tracts in Mathematics, Vol. II, World Scientific, 2006.

Department of Mathematics University of Notre Dame Notre Dame, IN 46556 mgursky@nd.edu 